\title{
Namenregister zu Band 79 und 80
}

A

Abraham yg 568, 80

107, 271 Abramowicz 80 68,

170 Accardi yg 384, 80272 Ackermann 79314 Adamantiades 80, 164 Addario 79 377, 80380

Adie 79421 Adler, F.H.S $44 \theta$ i(Bb.) Adler 80264 Aguiar 80180 Akesson 79, 544 Akksej ew 80 379 Alajmo 8066 Alajouanine 79559 Albrecht 80 294, 296 Albrich 80 82, 86, 94 Alcaino yg 309 Aleeva $<$ So 374 Allen yg 559 Altland 80 313, 314 Altmann 5o 309 Altschul yg 322 Amat 80364 Amberger 79396 Amemiya yg 550 d'Amigo So 165 Ammann 80304 d'Amore 80179 Amsler 7959 Amstislavskij 80286 Anderson 79 330, 80 68,

106 Andrade 80288 Andrasowa So 175 André-Thomas yg 318 Angelucci So 380 Antoniban yg 390, 392 Aoyama 80291 Apin So 74, 267 Archangelsky yg 361 Argañarez yg 317, 544,

< §o 173 Argüello yg 317 Arnold yg 389 Arruga 79 59, 61, 62 Asarova So 382 Ascher yg 569, 570 , So

379 Ashikaga 80292 Ask yg 401, So 366 Attiah yg 392 Attig 79568 Aubaret yg 59 Aubry 79 318 Aulamo 80178

Aust yg 579, So 396 Avizonis yg 558, So 297. 3OI $>3^{\circ} 2>36^{\circ}$

B

Baagoe So 162

Bab yg 395, So 162, 292,

301 Babonneix 79 315, 316 Bächtinger yg 82 Bader yg 399 Badot yg 52, 570, 80

158 Badt 79424 Bahr yg 417, 418 - Bailey yg 71 Baillart 79 55, 65, 318, 569, 80267 Baisi 80297 Bakarenkov yg 68 Balacco So 160 Balás 8097 Balcarek79 382, 80156 Ballod So 166 Balyeat 80293 Bannes So 273 Banu 80274 Baquis 80 371, 285 Barbel So 299

Barberousse yg 303 Bardancelli 80381 Barg So 372 Barison yg 318 Barkan So 168 Barletta yg 70 Baroni 80 2y8 Barraquer yg 61, 63 Barrenechea So 169 Barrière 79569 Bartels 79 29, 394, 80

$100,182,304,312,314$,

316, 376 Bartók yg 399 Batten yg 51 Baurmann yg 309, 412,

417. 418 Bauwens yg 568 Bayer yg 549 Beaumont 79 542, 80

72 Becchini So 178 Béclère 80180 Bedell 7972 Behr yg 67, 303, 382 Beigelmann yg 65, 569

Bellavia 80272 Belot So 377 Bender yg 65, 391

Bendescu yg 41 Berberoff So 379 Bercioux yg 83 Berg yg 563, 565 Berger 7972 Bergmeister 80229 Berliner yg 53, 379 Bermann 79556 Bernaud 80 292, 316 Bernstein 80186 Bertrand 79 390 Beruk yg 318 Beselin 80309 Bessemans So 290, 291 Best yg 457 Bey yg 392

Bhaduri 79559 Bielschowski yg 320,

580, So 392 Bienstock 80292 Bier 80289 Bieringer So 393 Bietti yg 72, 380, 384, 80299 Bing 79 65, 569 Birch-Hirschf eld yg

376, So 320 Bistis 80 250, 298 Bittorf yg 321 Black So 69 Blackma yg 562 Blank 80297 Blatt $7962,383,8071$, 
185, 186, 277 Blessig So 281 Blum yg 319 Blumenthal So 294 Böck 80287 Beegehold So 68 Boenheim yg 569 Boeve 80206 van Bogaert 79569 Bollack yg 306 Bolotina yg 316 Bolotta 79 321 Bol§akow 80160 Bömer 79 64, 80281 Boncinelli So 382 Bonnefon yg 61 Boquien yg 568 Borak So 386 Borel yg 89, 90 Bormacher 80 303, 315 Börner 79376 Boros 8090 Bothman yg 395, 80182 Botteri yg 562 Bourdon 79547

Namenregister zu Band 79 und 80.

417

Bourgignon 79545 Bouwdijk-Bastiaan-

se 79, 315 Brain 79309 Branbergen 80286 Braun 80 102, 293 Braunstein 79381 Brennscheidt 79329 Bretagne 79 307, 308 Brickner 79329 Brill 79 382, 570 Bruce 7970 Bruch 79321

Bruckner 79 568, 80

in, 182 Brumpt 5o 281 Bruno 80157 Bruusgaard 80282 Bucklers 79 567, 80

196, 200, 202 Bujadoux 80168 Bulac 79383 Bunge 79 5, 412 del Buono 79302 Buquis ¿?o 180 Burk 79 381, 395 Bursuk 80163 Busacca \#0 278 Bushnell 79388 Busscher 79317 Butler So 179 Butterfield 79319 Buytendifk 79548 Bychowski 79320

C Cailliau 79311 Cairns 79318 Calajanni 80301 CalengaSo 369, 372,373 Camauer 79314 Cameron 79 399, 558 Camison 80370 Canegham 79321 Cange 80281 Canneight So 290, 291

Carlotti 7954 Carmichael 79313 Casanovas So $3 \delta$ Casati 79566 Casini 80367 Caso 79 568, So 373 Caspari 80186 Castello 80378 Casteran So 183 Castronovo 79397 Cattaneo 79 377, 567, 80 278, 363, 377, 378 Caussée 79318 Cavegt 79568 Cavina So 163 Cazac-Averbuch 79560 Zeitachrift fur Augenheilkunde.

Cepurin 80, 368 Cerise 79302 Cerqueira So 298 Cetverikova 80 153,

168 Chafir 79302 Chalasnikow So 184 Chams 79 54, So 186 Chan 8045 Chantriot 80275 Charamis 79309 Charlin 79317 Chase 7959 Chiari 79568 Chichizola 79556 Chiser 79321 Cho 79558 Christophe 79310 Clairmont 79420 Clark 79539 Clarke So 112 Clausen 80167 , 173 Clavany 79389 Clay 79570 Clayborn 80292 Cluzet 79546 Cockaine 79395 Cocuzza 80 286 Coerper So 306 Colajanni 79 70, 542 Colle 80272 Collevati So 377 Collier 79 72, 569 Comberg 79 355, 414,

417, 426 (P.), 80180 Combiesco So 368 Comes 80160 Convález 80364 Cooper 79570 Coppez 79 54, So 375 Cosmettatos 79310 Coste So 179 Coudeau 79566 Courey 80305 Courtis So 176 Courtney 79562 Cowans So 75 Cozzoli 79567 Craig 79308 Cremer So 158 Cremieux 79558 Croci So 361 v. Csapody 79566 Csapody-Mossi So 183 Cucchia 79 546, 80 67, 183 Cucco So 163 Cuénod 80381 Curschmann 79416 Cushing 7971 Custodis 80 103, 320 (P.)

Cusumano 80 275, 369 Bd. 80. Heft 6 (Juni 1933).

Cuthberton 79 392,567 Cutler 79554

D Dahlfeld So 401 (Bb.) Dallos 79 560, 80 71, 90, 96 Darnel 79401 Daniels 79380 Danis So 168 Danzig 79547 Davidson 79315 Davies 79305 Davis 79 317, 540 Dedimos So 368 Deevy 79318 Defong 80362 Degeller 79320 Dejean 79 309 Delord So 167 Démétriadis 80271 Demme 79315 Denig So 165 Dentin 80304 Derer So 169, 177 Derkac 80367 Deutschmann 7960 Devé So 69 Dewer 79543 Dewulf 79317 Dickson 79318 Diez 79553 Dimitriu So 294 Ditrói 80 81, 84, 85, 97, 99. 3QI Doherty 7969 Dölberg So 309 Dolin 79311 Domenidó79 566, S074 Dömsödy 8098 Donahue 79554 Donin So 175 Dor 79 547, 560, So, 73 Dowman 79317 Downey 79 544, 568 Draganesco 79315 Dudinow 80287 Dufour 80 66, 68 Dufour-Baron So 72 Dufour-Donzelot 80 
67 Dufour-Goult 8074 Duke-Elder So 272 Dupuy-Dutemps 80

165 Dupuy d'Uby 80289 Durup 79551 Duschnitz So 158 Dusselmann 7972 Duynstee 79571 van Duyse 79571 Dvorak-Theobald So

280 Dyban 80 163, 380

27

$.4 * 8$

Namenregister zu Band 79 und 80.

Dymschitz yg 393 Dymsic yg 548

E. Edgerton yg 309 Edler 80365 Ehrenstein yg 540 Eliassow 80281 Elker 79302 Elschnig yg 560,567 ,

5o 168, 268, 391, 393,

400 (Bb.) Emmons yg 316 Enami yg 549 Endelman 79 66, 560,

568, 80367 Engel yg 304 Engelking 79 81, 397,

545, 55 Eppenstein 80372 Erben 80393 Erdös 8084 Erggelet So 166, 167 Erlandsson yg 69

ErlangerSo 175, 176 Erskoviz 80 165, 371 Espildora yg 56 Esser yg 400, 401 Esteban 79 544, 80

361 Evans 79566

$\Gamma$

Façon 79321 Falta 80 88, 377 Farago 80180 Farid 7959 Farkas yg 381, 393 Farnsworth yg 556

Fasselova So 277, 377 Favaloro 80 278, 376,

379 Favory yg 570 Fazakas yg 376, So 84,

87, 98, 301 Fehér 8097 Feigenbaum yg 376,

395 So 43 (Bb.) Feilchenfeld 80308 Fejér 80, 84, 301 Fernandez 80295 Ferree 79545 Ferro 80

304 Fessard 79551 Fesüs yg 396, 569, 80

159 Filatow So 165,166 ,

372 Finkenrath 80306 Finoff 80279 Fischel 79 548, 80 351,

379 Fischer F. (Wien) 79

$158,307,378,386,550$,

$8078,186,189,191$,

387

Fischer F. P. 80 260,

263, 272, 393 Fischer O. yg 313, 413,

569 Fischer-Ascher yg

377, 80279 Fisher 80170 Flandin 80183 Fleischer yg 51, 380,

543, 568, 80193,196 ,

198, 200, 202 Focosi 80 156, 285 Foerster, O. 80391 Fontana 79 384, 80 272,

364 Forberg yg 568 Ford 79302 Forknall yg 546 Foroni So 362, 367, 368,

37o Foshay 80365 Fossati yg, 320 Foster yg 569, So 180 Foucault yg 553 Fouché 79318 Fralik yg 50, 569 Franceschetti yg 88 ,

394, 557- 56o, 570,

80112 (P.), 182 Francois yg 317 Frank E. yg 310 Frazier 79309 Freudenthal yg 566,

So 130 Fribourg-Blanc yg

321 Frick 80180 Fried 79313 Friedberg yg 392 Friede So 72 Friedenwald yg 50,

So 263 Friedmann B. 80281 Friedrich yg 539 Friman-Dahl 79568 Frisbee 80295 Fritz yg 53,

So 265 Fröhlich yg 540 Fry 79305 Fuchs yg 393, So 190 Fujihira yg 66 Fujisawa So 283

Fülleborn 80287 
G

Gala yg 558, 565 Galewska 80375 Galewski So 164 Gallemaerts 80375 Gallenga So 292 Galli yg 550 Gallois yg 59, So 158,183

Galperson 80154 Garrahan 79559 Gasteiger yg 235, 538, 80 193, 199, 283, 303,

37a Gebb yg 467, So 1 Gebbert 80102 Gelard 79550 Genet 80173 Genkin 80298 Gennaro 79 562,80155 ,

364 Georgi 79313 Giani 80 -zy! Giannantoni 80 67,

260, 303 Gibson 80297 Gifford 80261 Gilbert yg, 50, 562 So

402 (Bb.) Gildemeister 80294 Gillessen 80 153, 370 Giorgio yg 548 Girandi 79317 Glasscheib 80293 Glees So 156 Glezerov 80360 Glimstedt 80295 Globus 79318 Gnad yg 393. 3/8 !79

Goar 79395 Goerlitz 79413 Goldenberg 80297 Goldf eder yg 93, So382 Goldschmidt So 303 Goldstein yg 69, 389,

558, 80204 Goldsteiner 7966 Gomez 80363 Gonin yg 60, 62, 86,

So 171, 302 Goo idale yg 318 Görlitz 80172 Gotoh yg 547 Gourfein 79321 Gourfein-Welt yg 89 ,

420 Goy 80160 Graf 79320 Graff 8073 Graham 79 541, 548 Granit yg 540, 541 Granström 80 365 Grassmann yg 544 Grenet 79315 Griffiths yg 554, 569 Grinker yg 70 Groenblad 79 382, 570 Groenouw 80394 Gross yg 544, So 186 v. Grósz yg 470, So 96 Gruhle 80306 Grunert 79 415 Grüngarten 80289

Namenregister zu Band 79 und 80.

419

, Grüter 79 477, 80 160, 169, 285, 374 Gryníelt 79388 Grzedzielski, 79, 68 Guaglio 80183 Gualdi 80 70, 185 Guglielmo 79568 Guillain 79 422, 80180 Guillaume 79 304, 307 Guillermin 7954 Guillery 7978 Guillot 3/80 375 Guiral 80265 Guist 7956 Günther 79397 Guthrie 79320 Guttmann 79305 Gutzeit 80187

$\mathrm{H}$

Haag 80293

Haas $79449,541,545$,

$546>553.80208$ Haase 80381 Haemmeli 7983 Haessig 79305 Hahn L. So 398 Haitz 7969

Halász 79 33, 80301 Halbertsma 79 66, 561 Hall 80289 Halle 79558 Hallervorden 79 320,

328 Halphen 79302 Hambresin 80 298, 375 Hamburg 79331 Hamburger 79 326, 402

Hammond 79541 Handmann 79381 Hanke 80159 Hannsen 79402 Hann sen 79381 Harrison

80170 Harrower 79555 Harston So 176 Hartinger 80 69, 70, 73 Hartleib 79 390, So 155

Hartmann 79 72, 317,

3i8. 558, 569 Hartung $7968,81,382$,

550, 570 Hashimoto 79548 Hassin 79315 Hauer 79311 Hauptmann So 276 Hayashi 80283

Hecht 79538 Heider 7957 Heine 79 51, 379, 40,

So 209 Heinemann So 371 Heinismann 79570

Heinonen 79 566, 570 Heinsius 79 52, 380 Heintz 79379 Heldt 79 389, 562 Hemmes 79 70, 71, 560 Hempel 79570 Hendricks 80314 Henneberg 79310 Hennessey So 287 Henninger 80205

Henschen 79 541, 568 Hensen 79314 Hentschel 80394 Herbert So 277 Hering 79538 Hermann

K. 79305 ,

So $130 \mathrm{v}$. Herrenschwand 79

223, 315 Hertel 79549 Herz 79401 Herzau 79 397, So 395 Hessberg 79 67, 77, 80 101, 102, 104, 300, 
$302,304,313,314$,

315 Hesse 79 247, 252, 382 Hestermann 7970 Heuer 79308 Heuss 80303 van Heuven 80317

Hildreth So 177 Hilgartner 79557 Hill 79562 Hipanova So 382 v. Hippel 79 52, 69, 71, 379.

$38^{\circ}$. 382, 569, 570, 80 318, 373 Hirose 80271 Hirsch 79 384, 80388 Hoeg 79 39i van der Hoeve 79558 ,

$57^{\circ}$ Hof 79567 vom Hofe 7981,511 ,

$55^{\circ} .569,80272,292$ Hoff 79 310, 315 Hoffmann R., 79 59,

391, So 124, 277 Hoffmann, S. I. 79315 Hoffmann W. 80 155,

158, 163, 175, 177 Hoîmarksrichter 79

553 Höglund 79316 Hohagen 80104 Holden So 282 Hollos 79394 Holloway 79306 Holm 79

330 Holmes 79310 Holmström So 71 Hondemer So 289

Horay 79 395, So 79, S7 Hornbostel 79539 Horniker 79570 Horowitz 79315 Horváth 80 80, 83

Hosono So 283 Hossmann 80394 Hough 8072 Houwer 79 378, 80318 Hövelmann So 316

Huber 80, 183, 304, 367 Hunter 79 548, 569

I

Ibsen 79388 Igersheimer 80169 Ighenti 79568 Ikezawa 7967 Imachi 80269 Imagawa 80283

v. Imre jun. 8082,88 ,

96, 97 Incze 8097 Inouye So 69 Isaak 80150 Itoh 7951 Iwanow So 164 Iwata 79307

$\mathrm{J}$

Jackson 79 564, So 66,

182 Jacobs 8074 Jacqueajud So 168 Jaensch'79 38o, 383,

580, 80 187, 394 Jäger 79304 Jahnke 79381 Jakubiak 80179 Jambon 79310 Janaevoska 80

381 Janson So 298 Jason 79568 Jatoba 80289 Jennings 79542 Jeremy 79566 Jess So 170 Jesse 80281 Johannsen 79543 John So 184 Johnson 79540 Jongh So 270 Jordan 79377 Joyeux 80

289 Judkevic So 380 Juhasz-Schäffer 80

298, 321 Junes 80 164, 382 Jung So 101 Jungraw 79368 Junius 79 67, 568, 569,

So 106, 365 Just Tiscornia 80280 Justin 80158

$27^{*}$

420

Namenregister zu Band 79 und 80.

K

Kafka 80 189, 191 Kahn 79547 Kahoun 7967 Kalaänikow 79546 Kalfa 79 547, 80270 Kalt 80 361 Kaminski 79377 Kandasvany 80288 Kanerva 80285 Kankrow So 168, 287 Kanyó 80 91, 278, 290,

379 Kaplan 79319 Karbowsky So 362 Karger, S. 80403 (P.) Kartagener 79568 Katona 79543 Katznelson 79313 Kawamura So 283 Kayser 80364 Kazdan 8071 Kazlanskos So 302 Kern 79 21 Kernohan 79308 Kessler 8076 Kesten 80374 Kestenbaum 79 405,

80389 Kiel So 289 Kikai 79380 Killen 7932 Kimel 80382 Kimura 80283 Kisch So 264 Kiso

79383 Kitamura 79568 Kiyosawa 80304 Klainguti 79 82, 569 Klauber 79 546, 80 154,

166, 168 Kleiber 79383 Klein So 81, 83, 88, 93,

152 Kleiner 79 306, 8077 Kleinmann 79568 Klemmer 79570 Klossowsky 79568 Klövekorn

80179 Knapp 79 52, 80154 Knobloch 79 560, 80

178 Knodel 79569 Knutter 80279 Ko ch-K a wali kowa So

155 Kochmann 79517 Koffka 79555 Köhler, G. So 207 Kojima 7952 Kolén So 165, 383

Komiyama 80281 Kondo 79 542, 544 Kopp 80362 Körber So 315 
Körner 79402 Kostoulas 79562 v. Köteles 80159 Kotlj arewskaja 79

65, 569 Kotrnetz 79384 Koyanagi 79 51, 57, 69 Krabbe 79311 Krachmanikow 7966, 556, 564, 80381 Krakov So 165 Kramer 79 579, So 66,

188 Krantz 80292 Krassó 79 172, 378, 382,

394, 80371 Kraupa 79565 Krause, A. C. 80259 Krauss 80102 Kravitz 79310 Kravkow 79

543, 549 Kredbova 79, 394, 560 v. Krehl 80319 Kreibig 79 52, 380, 383,

8023 Kreiker 79377,8083 ,

$85,87,88,89,93,95$,

98, 184, 289, 383 Kreutz 7966 Kroll 79306 Kron 79319 Kronfeld 80264 Krückmann So

101,169 Kruglov 80178 Krynov 80382 Kubik 79 320, 378, So

$3^{\circ 0} .394$ Kuchner 79379 Kufs 79 568, 569 Kukán So 94 Kümmell 79419 Kunii 79542 Kuniya

7967 Kunz 79, 379, So 152 Kurz 79 52, 59, 567 Kutzinski 79424 Kyanagi 79383 Kyander 79

395, 557 Kyrieleis 79, 58, 302,

312

L Lacarrère 80170 Ladekarl 80281 Lagrange 7957 Lamma 79562 Landegger 79378

Landsbergienié 80

308 Lange 80290 Langguth So 157, 176 Langmann 79419 Larsson 80 172, 268 Lasarew 79548

Laschi 79304 László 8095

Lauber 79 545, 80 69,

180, $402(\mathrm{Bb})$ Lauterstein 79370 ,

So 58, 362 Law 79 66, 399 Lazarescu 79 315, 320,

393- 56o Learmonth 79308 Lebensohn 8068 Lees-Sinskovits 79

384 Lehrfeld 80 108, 367 Lenz 79559 Leoberas So 362 Leonardi 79543, So 300 Lépine 79313

Leschke 80 297, 298 Lesné 79568 Levin So 303 Levine 80160 Levinsohn 79 564, So

182 Lev ison 79570 Levy 79567 Lewi M. 79180 Lewis 80 73, 289 Lhermitte 79315 Lidström

So $153 \mathrm{v}$. Liebermann 8078 ,

86 Lièvre 79568 Li jo So 363 Lillie 79 308, So 298 Limper 79544 Lindau 7972 Lindemeyer So

197 Lindgreen 79558 Lindner 79 61, 63, 191,

405, 408, 579, 80171 ,

172, 311, 387 Lindsay 80366 Linksz 79 546, 80 81,

91, 92, 98 Liorber 80 178, 382 Lipschütz 8063 List 79 306, 309 Litauer So 164, 375 Litinskij 79

546, 547 Little 79388 Ljubin 7966 Lobeck 79 562, 80 263,

275 Lobel So 172 Lockau 80294 Loddoni 79392 Löffler So 312 Löhlein 79 53, 59, 524,

80 166, 167 Lombroso So 279, 366,

379 Looft 79569 Loos 79557 Lopez 7961 Lopez Lacarrière 79

545

Namentegister zu Band 79 und 80.

42I

Löwenstein 79 68, 320, 378, 389, So 165, 179,

383 Lowery 79539 Lubarsch 79580 Luedde 80184 Lukowskij 80157 Lumban 80378

Lundquist 7969 Lundsgaard 80163 Lunz 79 540, 80156 v. Luzsa 80155 Lyster 7968

$\mathrm{M}$

Machener 80105 Maggiore 8072 Magitot E0 262 Mahoney 80298 Mainzer \#0 366 Majima 80 157 Makay 80259 Malaguti So 286 Malbran 79 304, 545 Malkin 80175 Mandl 79315 Manes 79304 Mann 79321 Mans 79 414, So 320 (P.) Marchesani 79 67, 305, 382, 564, 570, So 181, 
184, I97, 20I, 202, 307

Marghrati 80381 Margotta 79 82, 566 Marin-Amat 79 557,

80179 Marinesco 79553 Mariotti 79304 Markus So 380 Mårquez 80 72, 286,309 Marshall 79

69 Martel 79 304, 307 Martin 79315 Martini 80293 Martius So 368 Martyn 80293 Marx 80

156, 206 di Marzio Salvatori

80178 Mast 79540 Mathewson 80363 Matsuda 79548 Matsuoka So 183 Ma was 79 72, 569

Mayedo 79 568, So 297 Mayer Th. 80287 Mayer-Hillebrand 79

554 Mayon 80 366, 370, 374 Mc Allister 79556 Mc Callau 80380 Mc Coe 7952 Mc Crae 79

65 Mc Dowall So 180 Mc Ilroy 79 557, 558

Mc Kee 7959

Mc Lacklan 79315

Meerhoff 79 390, So

373. 374 Meesmann 79 381, 545,

So 266, 269 Meili 79554 Meisner 7950 MeissnerS948, 180,308 Meller I. 79 63, 95, 110 , 128, 408, 80208 (P.),

280, 300 Meller, R. 80280 Mendoza 80170 Menninger- Lerchen-

thal 79390 Mergitot de Treigny

80176 Meriel 79310 Merrill So 45 Messing 79396 Mets 79400 Metzger 79541 Mewes 79317

Meyer 79 569, 80169 ,

398 Meyer-Steineg So 360 Michael 80261 Michaelson 80182 Michail So 278, 286,

294. 36 $.368,379$ Michniewicz 80276 Michon 79 307, 308 Mickewicz So 280, 375 Miescher

79378 Mieth 7967 Miget 79 315, 316 Mikaeljan 80 275, 279,

380 Miklós 80 154, 276, 370 Miles 79542 Milewska So 153 Milian 79315 Mills 80293 Milner

79 569, 80180 Minakawa 7972 Mirimanoff 79 397,570 Misugi So 283 Mitamura 80283

Miyagawa 80283 Miyashita So 182 Miyazav ¿a 80283 Mohr 79 70, 383 Mollart 79313 Moller

80376 Monbrun 79 304, 307 de Monchy 79570 Mönckeberg 79 78, 307 Monje 79547

Montanelli 79546 Moore 79 62, 558, 80180 Morax 79 52, 80275 Moreau 79568 Morelli 79

67 , So 159 ,

264,372

Moretti 79 52, 567 Morgan 79559 Moroz 79391 Morton So 184 Motais 80289 Motegi 79 375, 80378 Motolesi 7968 Much 8070 Mühlens 80 289, 297 Müller, E. 80184 Müller, G. E. 79

540, 542 Müller H. 79 64, 375,

395 Müller, H. K. 79 557,

So 113, 186, 208 (P.),

267, 380 Munch 79566 Munn 79556 Muntsch 80298 Mura 80287 Muredder 7959 Musial 80

147254 Mylius 79 383, So 170,

289

N Nabe 79517 Nagayo 80283 Nagy So 94, 277, 378 Nakamura 79 91, So

161, 297 Narayuna 79561 Naròg So 176 Nass 79555 Natanson So 299 Nauck 80287 Nayar 80

288 Neame 7952 zur Nedden 7964 Nègre 7964 Neiding So 297 Neuhann 80315 Neuhaus 79

554 Nicolato 79 375, So 372 Nicolic 80183 Niessl v. Mayendorf 79

310 Niimi 80 178, 382 Nishibe So 283 Nobl 79569 Nogue 79554 Nonaka So 260 Nonne 79312

Noothoven van Goor

79307 Nordmann 79304

Nowak 79561 Nyulassy 80 164, 167

0 
O’Brien 80 72, 261 Ochi So 278, 378 Odell 79314 Odnasewa So 178 Oeser 79553 Oestreicher 80374

422 Namenregister zu Band 79 und 80.

Oguchi 79, 72, 393, 567,

569 Ohm 80 313, 315 Okabe 80301 Oláh 80 89, 152, 319,

$37^{\circ} .376$ Olin 80280 Olitzki 80279 Ollendorf $\gamma$ g 567 Ollendorff 80392 Oloff 79 320, 545

Oltmann $\gamma \mathrm{g} 64,567$,

80302 Oneto 79 68, 80287 Onken E0 375, 376 Oppolzer $\gamma$ g 576 Orel $\gamma$ g 558 Orgaz 7965

Ornsteen $\gamma \mathrm{g} 569$ Ortloph $\gamma \mathrm{g} 329$ Orís Llorca 79378 Ostroumow 80306 O’ Sullivan 80177 Ovio

(So 67 Owen \#0 287 Ozimin 80175

$\mathrm{P}$

Pal 7956 Pallarés $\gamma$ g 67, 68 Pandolfi $\gamma$ g 565 Panico 79565 Pansdorf $\gamma$ g 549 Papolczy $\gamma$ g 376, 379 ,

558, So 377 Parker $\gamma \mathrm{g} 50,569,80$

298 Pascal 79 545, So 72, 74 Pascalin $\gamma$ g 59 Pascheff 80368 Passow 79 545, 80 194,

203 Patry $\gamma g$ 89 Paul 79 410, 414 Pavia $\gamma g$ 62, 72, 390,

547 Pavlov 80281 Payenville $\gamma \mathrm{g} 311$ Pellathy 79 53, 80 86,

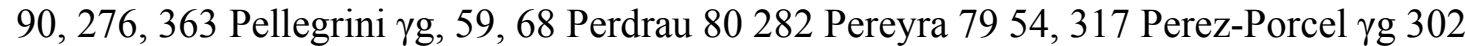

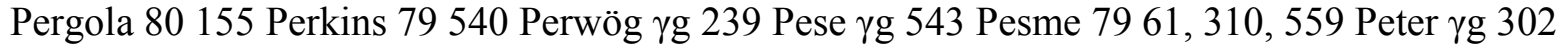

Peters $\gamma \mathrm{g} 1,94$ (P.),

376, 416, 80 365, 381 Petow 80293 Petres So 85, 93 de Petri 80274

Petrignani $\gamma \mathrm{g} 309$,

570, 80291 Petroni So 381 Petrowic $\gamma \mathrm{g}$ 321, 396,

559 Petrunja 80 164, 375 Peyer 80309 Pfahler 79 308, 80180 v. Pflugk 79 401, 80

184, 186 Pick $\gamma g 424$ Pieck 79379 Pierce 80263 Pikler 79543 Pillat $\gamma g 94$ (P·). 200,

327. 377. 80188,189 ,

$191,274,368,385,389$,

39i, 399 Pincus 80103 Pines 80 164, 383 Piquero 80380 Pires $\gamma g$ 312, 313 Planques $\gamma g 310$

Plomann 80362 Podestá 79542 Pokrovskij 80374 Polack $\gamma$ g 542 Poleff So 292 Pollak $\gamma \mathrm{g} 378$

Pollano 79312 Pollarés $\gamma g 310$ Ponomarev $\gamma g 562$ Ponthus 79546 Poos $\gamma g$ 68, 78, 80, 382,

570, 80 105, 266, 270 Popov $\gamma \mathrm{g} 564$ Popova 80276 Popovic 7951 Poppinga $\gamma g 553$ Porru 7970

Post 80176 Prangen 80186 Prausnitz 79401 Pressburger $\gamma g 379$ Prieur 79 567, Proctor 80279

Proksch 80 71, 362 Proppe 79314 Protzer 80201 Pta§nik 79 566, 80 165,

383 '.

Puntigam $\gamma \mathrm{g} 315$

Q

Quint 79 66, So 302

$\mathrm{R}$

de Raadt 80397 Rabinovic $\gamma \mathrm{g}$ 65, So

294 Rabinowitsch $\gamma g 59$ Rachmann So 156 Rados $\gamma g 69$ Rail 8071 Ralson So 193 Ramaswamy

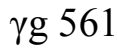

Rambar So 298 Rand $\gamma g 545$ Rappoport yg 318 Rasquin So 375 Ratner 80374 Rauh 79 307, 80 259,303 Raverdino 80172 Redslob 79 60, 80186 Reese 7970 Reible 80156 Reichling 79566 Reilly yg 558 Reinhards 80308 Reis So 265 Reiss 80261 Reitsch 79 374, 80 393, 395 Reuling 80301 Reuter 79320 Revész $\gamma$ g 554 Ridley So 282 Riedl $\gamma g$ 287, So 301 Riegel $\gamma g$ 433 Rieger $\gamma g$ 377, So 76, 
279, 379 Riehm $\gamma \mathrm{g}$ 50, 51, 80 197,

291, 294, 364 Rifat $\gamma$ g 308 Ringelhan 79560 Rinkel 80293 Risak 80388 Riser 79310 Rizzo 80

285 Robertson So 259 Rochat $\gamma \mathrm{g} 72,318,80$

69. 73 Roche So 261 Rochon-Duvignaud

yg 308, So 180 Roger $\gamma g$ 558, So 381 Roggenkämper 7964,

80104 Rohracker $\gamma \mathrm{g} 555$ Rohrschneider 79 91,

378, 80 205, 294 Rolett 80 303, 380 Roscin $\gamma \mathrm{g}$ 69, 383 Rosenbaum 79391 Rosenberg $\gamma \mathrm{g} 59$

Rosenblatt 80372 Rosengren So 363 Rosenstein $\gamma \mathrm{g} 396$ Ross 79 395, 557. 558 Rossi $\gamma \mathrm{g}$ 65, 316, 562 ,

80381 Rössle $\gamma$ g 376 Rössler 79 545, 8074.75 Rosso-Fratasi 79302 Rostohar $\gamma$ g 554 Rothmann yg 565 Rötth So 81, 83, 87, 91,

94- 95. 97. 277, 279,

292, 377- 379 Rowe 80293 Rowland $\gamma \mathrm{g} 306$

Namenregister zu Band 79 und 80.

423

Roy 80163 Ruata 80382 Rubbrecht yg 63, 64, 80167 Rubert 80381 Ruge \#0 314 Rugg-Gunn 8070 Rüssel 79554

S Sabadeanu 80290 Sachnenko yg 556, 564 Sachs M. yg 403 Saethre 79397 Safar yg 404, 578, 80

312, 374 Sala \#0 66 Salgo yg 306 Salinger 80179 Salit < So 261 Sallmann yg 208, 378, 407, 80 71, 181, 342 Salom 79570 Salus 79 58, 63, 91 Saloati yg 315, 80 175,

374 Salzer 79395 Samojlow yg 50 Sander-Larsen 80362 Sani 79318 Sanna yg 302, \#0 67

Santonastaso 80 361,

368, 376 Sarbó yg 313 Sarkinsjanc \#0 164 Sasaki 80260 Satanowsky yg 59, 71 Sato \#0 283

Sattler 80304 Savelev 80168 Savin 79 65, 69, 569,

80283 Sazato 80283 Scales \#0 298 Scalinci yg 401, So 295 Scarlett yg 67 Schachter So 360

Schaeffer yg 315 Schäfer yg 543, So 309 Schaffer yg 568 Schaly 79307 Scharapow yg 312, 318

Scheerer yg 57,383 ,

396,568,50194,200,202 Scheffer 80152 Scheuermann So 304 Schick yg 315 Schieck 79 66, 94, 80

in, 160, 200, 294, 365 Schiff-Wertheimer

79 65, 569, 80158 Schilling 80 no Schlaepfer So 301 Schmelzer 80 160, 198,

$275,292,295,356,369$,

$37 \mathrm{i}$

Schmerl 79545,50261 Schmidt, H. So 86, 156,

257 Schmidt, K. yg 427,

449, 80 69, 179, 260 Schmite yg 310 Schneider, K. 80 90,

92, 176 Schneider, R. yg 265,

320, 362 Schnurmann 80379 Schnyder yg 82, 85,

80179 Schoeler 80303 Scholtyssek 80 394,

396 Scholtz 80293 Schönberg 7964 Schor yg 570 Schornstein 80234 Schroder C. 7958

Schroderus yg 58 Schuback yg 72 Schubert 79313 Schulte yg 555 Schulz 79391 Schürich yg

420 Schuurmann 80278 Schwartz So 296 Schwarz Th. yg 59 Seckbach 80158 Sedan So 179

Seefelder yg 212, 378,

386, 399, 80 178, 192, 
195, 200, 203 Seeliger 80399 Sefic 80158 Sehlstedt So 280 Seidel yg 569, So 262, 361 Sena yg 59 Sevecuk 80179 Shannon 79 308, 309 Shapira 80394 Shostid yg 321 Shimoyama 79394 Shindo yg 70 Shoji yg .68 Shope So 268 Sigwald yg 422 Simone So 260 Simson yg 65 Singer, J. 8085 Sivelli 79559 Sjögren yg 316 Slavik 80168 Smaltino So 157 Smith, W. A. yg 317 Snoke yg 570 Sobby 7967 Sokolansky yg 568 Sokolova yg 391 Solafikowa 79566 Solotniskij 80 165, 371 Somogyi So 84 Sondermann yg 386 , 387, 388, 80 167, 266 Sönning 80317 „, Sorsby 79 67, 395, 558, 560, 80 182, 183 Sosmann yg 568 Souques yg 390 Sourdille $7960,63,72$, 318, 80171 Spackmann yg 308 Spatz yg 397 Srinivasan 79566 Stallard 80180 Stammers 79 308, 309 Staánik 80180 Stefanova 80379 Stein 79 68, 580 (P.), 80 162, 395 Steiner yg 314, 562 Stelling 80 153, 382 Stern, H. yg 361, So 76, 188 Stevensen yg 392, 567 Stewart 80259 Stieda 79318 Stiefbold 80 274, 372 Stilo yg 384, So 260, 375 Stine 80398 Stock 80178 Stocker 7984 Stoewer 80 314, 315 Stooss yg 568 Strandberg 79382 Strandell yg 68 Strebel So 161, 293 Strehl 80309 Streiff yg 560, 561 Strisower yg 172 Stuhl 7970 Styskalowa yg 390 Süchting 79 69, 381 Suda 80185 Sudzuki yg 58 Svensson 79565 Swab yg 561, So 298 Sweerts yg 568 Szabó 79 376, 380, 8080 Szász So 87, 92, 260 Székely yg 272, 386 v. Szily yg 79- 80, 380 Szymanowski yg 396, $80183,279,308$

Tagami yg 50 Takacirowa 79302 Takamatsu 80378 Takamura 80178 Takatsuji 79546 Takeo yg 50 Tallo So 295 Tamiya 80283 Tamura 79 564, 80 181,

269 Tänäsescu yg 320

424

-Namenregister zu Band 79 und 80.

Tanturri 79 392, 567 Tecce 79558 Teleky 80307 Teno 79318 Teräskeli 80278 Ter-Organesjan 79

302 Terrien $7963,389,422$ Terson 80156 Thiel 79 66, 308, So 179,

393 Thier 80307 Thies 80, 303, 393 Thim 80 277, 366 Thomsen 80182 Thomson 79401

Thorner-Spanuth 80

73 Thygeson 5o 279, 379 Tirelli 79 59, 558 Tobeck 80361 Tobgy 79 67, So 166 Tobler 79554

Torbin So 373 de la Torre 80288 Torvella 80288 Tó.th So 84, 89, 97, 361 Towbin 80141 Town So 295 Toyama 79318 Trabut So 289 Traumann 80 197, 204 Treacher-Collins 79

7i Trénel 79567 Trettenero 80186 Tria 80260 Tripodi 79316 Tron 79565 Trubek 7969

Tscheglowa 79558 Tschemolossow 79

321, 396, 559 Tschermak-Seyse-

negg 79538 Tschernyscheff 79

3 i8 Tsiminakis 79320 Tsunekawa 7952 Tyler So 279

$\mathrm{U}$

Ullrich 79397 Undelt 80307 Urban 79540 Urbanek 79 145, 575,

So 280, 311 Urrets 7957

Vail 79 302, 308, 389 Vajda 80 88, 89 Valda 80183 Valenzuela So 363 Valière 7952

Valière-VialeixSo 167 Vallée 7972 Vampré 79309 Vancea 79 52, 54, 312, 391, 80 261, 294, 377 Vannas 79 93, 379 Vasquez 7972 Veasy 79392 Veil 79 308, 389 Vejdowskij 80168 Velhagenjun. So 261 Venco 80265 Verbitzkij 8066 Verdaguer 7970 Verhoeff 79 53, 65 
Verrijp 79563 Vestergaard 79546 Viallefont 79310 Villard 7959 Villaret 80158 Villey 79553 Vincent 79318 Vincka 80290 Vita So 70

Vito 79 67, 80 295, 379 Vogelsang 79 78, 433 Vogt 79 82, 84, S5, 86, 87, 88, 320, 381, 393, 394, 426, 560, 80 168, 286, 298, 300, 366 Vollmer 79568 Volovic 79 65, 569 Vormann 79 394, 396 ,

559 Vuéicevic 80287

$\mathrm{W}$

Waardenburg 7966 , 564, 566, 570 Wachstein 79313 Wagner 80 154, 370 Wakely 79318 Walker 79304 Warschawski 80164 , 382 Wascowitz 79 66, 568 Watanabe So 294 Wätzold 807 van Waveren 80302 Weber 7981 Weekers 80 271, 375 Wegner 7998 (P.), 532, 80360 Weil 79 382, 570 Weill 79 304, 394, 560 Weinberg 79318 Weingott 80163 Weinstein So 93,96 ,

264 Weiss 79 65, 569, 80156 v. Weizsäcker 80307 Wendrovic 79 319, 320, 568 Wentworth 79546 Werke 7951

Werner 79545 Wessely 79 63, 393, $80172,196,200,202$,

376 Weve $7961,314,380$, 394, $8099,173,268$, 302 Wexler $7966,389,558$,

80204 Weymann 80 71, 176 Whalman So 159 Wheeler 79 540, 568 Whitwell 8069 Wibaut 79 $65,563,565$,

80159 Wibo 79317 Wieczorek So 276 Wiesli 79 378, So 157 Wilcoz 80184 Wilczek 79394 Wilder 79 313, 329 Wilenski 80141 Willi 79569 Williams 80382 Williamson-Noble 80 282 Willrich 79545 Wilson 80 166, 379 Winkler 79 318, 327 Winter 79307 Winther 79319 Wirz 79382 Wittich So 195 Wolf 79548 Wolff 79 66, 67, 305, 80 270, 303 Wölfflin 7967 Woods 79 306, So 294 Worms 79 54, So 185 Worster-Drought 79 3 i8 Wostry So 280 Wright 80366 Wulkow So 153, 243

Yagües 7959 Yamada 80160 Yamaga 79396 York 79320 Yoshida 79376 Youriéwitsch 79 556 Yow 7967 Yudkin 7953

$\mathrm{Z}$

Zauli So 177, 274, 370 Zavalevskaja 80 153,

1. 168 Zeemann 7952

58

70 ,

71- 395. 542 Zeitler 79412 Zingabe So 364 Zoldan 79392

560 Zondek

H. 80207 